\title{
Comparing pharmacologic mechanism of action for the vesicular monoamine transporter 2 (VMAT2) inhibitors valbenazine and deutetrabenazine in treating tardive dyskinesia: does one have advantages over the other?
}

\author{
Stephen M. Stahl
}

The two approved treatments for tardive dyskinesia both inhibit the vesicular
monoamine transporter type 2 (VMAT2) yet have pharmacologic properties that
distinguish one from the other. Knowing these differences may help optimize which
treatment to select for individual patients.

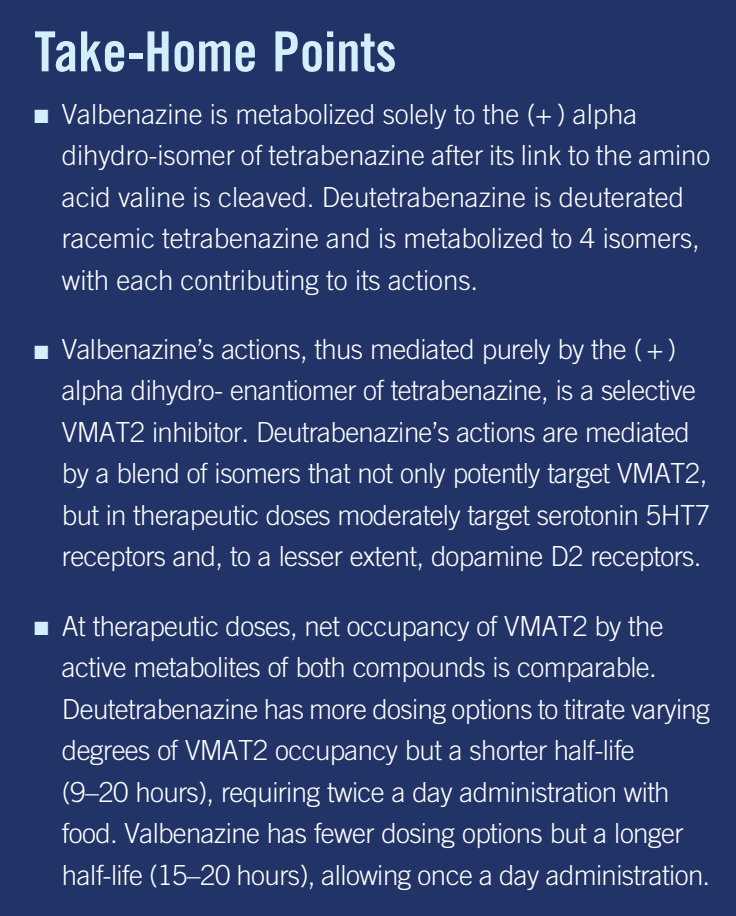

Introduction

The introduction of 2 different treatments has revitalized interest in tardive dyskinesia (TD), especially how to recognize and manage it. ${ }^{1-12}$ Both therapeutic options-valbenazine and deutetrabenazine-are based on tetrabenazine pharmacology. By blocking the vesicular monoamine transporter type 2 (VMAT2) in their own unique ways, both improve the involuntary movements of TD due to reducing dopamine availability at hypothetically supersensitive D2 dopamine receptors in the motor striatum. ${ }^{1,2,13}$ Both treatments are now first line recommendations for TD according to recently published guidelines and expert opinion, $^{3,4,12}$ but without any recommendation about whether one should be preferred over the other in any specific patient subtype. Thus, the question is left unanswered as to whether one way of blocking VMAT2 better than another for different patients.

Unfortunately, no head-to-head trials of valbenazine versus deutetrabenazine have been conducted to answer this question, and none is likely ever to be done. So, in the absence of such trials, is it possible for the two to be compared? Comparisons have already been made in several review articles by looking at how each drug performs against placebo in separate clinical trials. $^{5-12}$ The differences in safety and efficacy of the two new treatments studied in those trials have been extensively discussed elsewhere, ${ }^{5-12}$ with the two tardive dyskinesia treatments showing more similarities than differences. Here we look at these two agents in another way; namely, by contrasting their individual pharmacologic mechanisms of action. We have employed this strategy to generate discussion 


\section{BRAINSTORMS-Clinical Neuroscience Update}

regarding whether differential pharmacology could provide advantages or disadvantages of one of these new treatments over the other for different patient subgroups. We also hope this mechanism of action perspective might stimulate clinical trials and publications of clinical observations for how these pharmacologic differences may translate into how choosing the best agent and dose for an individual TD patient.

\section{Valbenazine Is a Solo}

Figure 1 shows how both VMAT2 inhibitors are metabolized into biologically active agents that mediate the known pharmacologic actions of the parent compounds. Valbenazine is the $(+)$ alpha isomer of tetrabenazine linked to the amino acid valine for slow release upon cleavage and then rapid metabolism to only one active moiety, namely $(+)$ alpha dihydrotetrabenazine (Figure 1)..$^{8-12}$ This active metabolite is the most potent inhibitor of VMAT2 of any tetrabenazine isomer, as well as the most selective, with only known actions on VMAT2 (Table 1). ${ }^{14}$ Thus, valbenazine's pharmacology is mediated by a "solo performance" of $(+)$ alpha dihydrotetrabenazine (Figures 1-3). The $(+)$ alpha isomer is then inactivated by CYP2D6 (Figure 1).

\section{Deutetrabenazine Is a Medley}

On the other hand, deutetrabenazine is the deuterated form of racemic (+/-) tetrabenazine..$^{5-7,11,12}$ While deutetrabenazine active isomers retain the VMAT2 affinities of the non-deuterated isomers, deuteration slows down metabolism, prolongs the half-life, reduces metabolic variability and lessens peak plasma drug levels of active isomers, thus allowing less frequent daily dosing and potentially an improved side effect profile compared to non-deuterated tetrabenazine. ${ }^{11,15,16}$ Tetrabenazine itself is approved for the treatment of Huntington's disease but not for TD, whereas deutetrabenazine is approved both for

Figure 1. Pharmacologic properties of the active metabolites of the vesicular monoamine transporter 2 (VMAT2) inhibitors valbenazine and deutetrabenazine. Valbenazine is converted into just one active metabolite, $(+)$ alpha dihydrotetrabenazine, which is present at high concentrations in the plasma, has high VMAT2 affinity and high VMAT2 occupancy.

Deutetrabenazine is metabolized by carbonyl reductase to 4 active dihydro metabolites. Two of these (the $(+)$ alpha and the $(+)$ beta dihydro isomers) have high affinity for VMAT2, but only the $(+)$ beta isomer is present in a sufficiently high amount to generate the VMAT2 occupancy required for therapeutic actions in tardive dyskinesia. Two other isomers, the (-) alpha and the $(-)$ beta dihydro isomers, have affinities for other receptors, but only the (-) beta is present at sufficiently high plasma concentrations to have moderate serotonin 5HT7 and low dopamine D2 occupancies. All active isomers are inactivated by CYP450 2D6 


\section{BRAINSTORMS-Clinical Neuroscience Update}

\begin{tabular}{|c|c|c|c|c|}
\hline Target & (-)-beta-HTBZ & (-)-alpha-HTBZ & (+)-beta-HTBZ & (+)-alpha-HTBZ \\
\hline VMAT2 & 690 & 250 & 10 & 4.2 \\
\hline Dopamine $\mathrm{D}_{2}$ & 53 & 180 & $>1000$ & $>1000$ \\
\hline Serotonin $5-\mathrm{HT}_{1 \mathrm{~A}}$ & $>1000$ & 750 & $>1000$ & $>1000$ \\
\hline Serotonin $5-\mathrm{HT}_{2 \mathrm{~B}}$ & 460 & 600 & $>1000$ & $>1000$ \\
\hline Serotonin $5-\mathrm{HT}_{7}$ & 6 & 71 & 970 & $>1000$ \\
\hline$\alpha 1$-Adrenergic & 980 & $>1000$ & $>1000$ & $>1000$ \\
\hline$\alpha 2$-Adrenergic & 220 & $>1000$ & $>1000$ & $>1000$ \\
\hline
\end{tabular}

Shown here are the binding affinities for the four active isomers of tetrabenazine taken from Grigoriadis et al ${ }_{1}^{14}$ assuming that these binding properties are also the same for the deuterated isomers.

Figure 2. Comparison of estimated steady state Cmax values of deutetrabenazine and valbenazine active isomers. Active isomers of 2 unrelated doses of valbenazine and deutetrabenazine within their therapeutic dosing ranges were determined at maximum plasma drug level (Cmax) following administration in separate studies. ${ }^{16-20}$ Valbenazine has only 1 active isomer, and deutetrabenazine has 4 , with the $(-)$ alpha and the $(+)$ beta isomers present in sufficient amounts to exert pharmacologic activity. Active isomers of deutetrabenazine and valbenazine cannot be directly compared. They are shown here to demonstrate the relative contributions of the different metabolites to the therapeutic plasma levels.

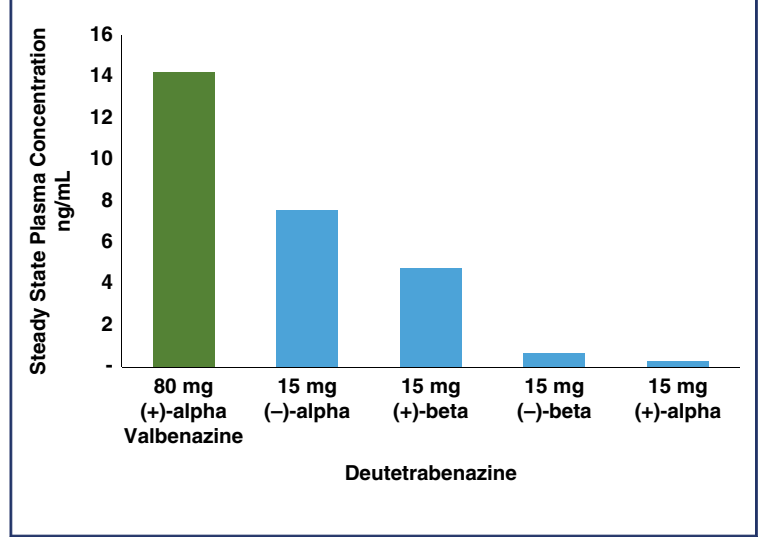

Huntington's disease and for TD. ${ }^{11,12}$ Pharmacokinetic differences between deuterated and nondeuterated tetrabenazine have been discussed elsewhere, ${ }^{5-7,11-16}$ but here we tackle instead comparisons of pharmacological mechanisms of action between the two agents specifically approved for TD, namely deutetrabenazine and valbenazine.
In that regard, if valbenazine is a solo performance by a single active metabolite, then deutetrabenazine is a medley, actually a quartet. Deutetrabenazine has 4 active deuterated metabolites (Figure 1), two of which act at VMAT2 and two of which act at other receptors (Figure 1 and Table 1). ${ }^{5-7,11,12,14}$ Specifically, the $(+)$ beta and the $(+)$ alpha deuterated dihydro metabolites combine to mediate the VMAT2 inhibition of deutetrabenazine (Table 1). Although the $(+)$ alpha form is the most potent inhibitor of VMAT2 (Table 1), unlike valbenazine, its concentration in the blood is quite low (Figure 2). ${ }^{17}$ For deutetrabenazine, instead it is the $(+)$ beta form of deuterated tetrabenazine, a less potent VMAT2 inhibitor (Table 1), ${ }^{14}$ but in much higher concentrations in the blood (Figure 2), ${ }^{17}$ that is the principle mediator of VMAT2 inhibition.

This duet of $(+)$ dihydro isomer metabolites of deutetrabenazine acting on VMAT2 is joined by a second duet of metabolites, namely the (-) alpha and the (-) beta forms of deuterated dihydrotetrabenazine (Figures 1 and 2). ${ }^{17}$ Neither (-) isomer blocks VMAT2, but both have significant affinities for 5HT7 serotonin receptors, moderate affinities for D2 dopamine receptors, and low affinities for other neurotransmitter receptors (Table 1). ${ }^{14}$ The predominant (-) dihydro isomer in the blood is the (-) alpha form (Figures 1 and 2), with relatively little contribution by the (-) beta isomer (Figures 1 and 2). ${ }^{17}$ Thus, the (-) alpha isomer mediates the pharmacologic actions of deutetrabenazine at various neurotransmitter receptors (Table 1).

All active metabolite isomers of deutetrabenazine are further metabolized to inactive products by CYP450 2D6 (Figure 1). 


\section{BRAINSTORMS - Clinical Neuroscience Update}

Figure 3. Comparison of estimated Cmax values of isomers of valbenazine and deutetrabenazine active at VMAT2 over therapeutic dosing ranges. Maximum concentrations of the metabolites active at VMAT2 for both valbenazine $[(+)$ alpha dihydrotetrabenazine] and deutetrabenazine [combination of both $(+)$ alpha and $(+)$ beta dihydro deuterated tetrabenazine] are shown from separate studies. ${ }^{16-20}$ Valbenazine doses are administered once daily, and the deutetrabenazine doses are administered twice a day. Levels of the different metabolites for valbenazine and deutetrabenazine cannot be directly compared. They are shown here to demonstrate the basis for calculations to convert these plasma drug levels into estimates of VMAT2 occupancy so those can be compared.

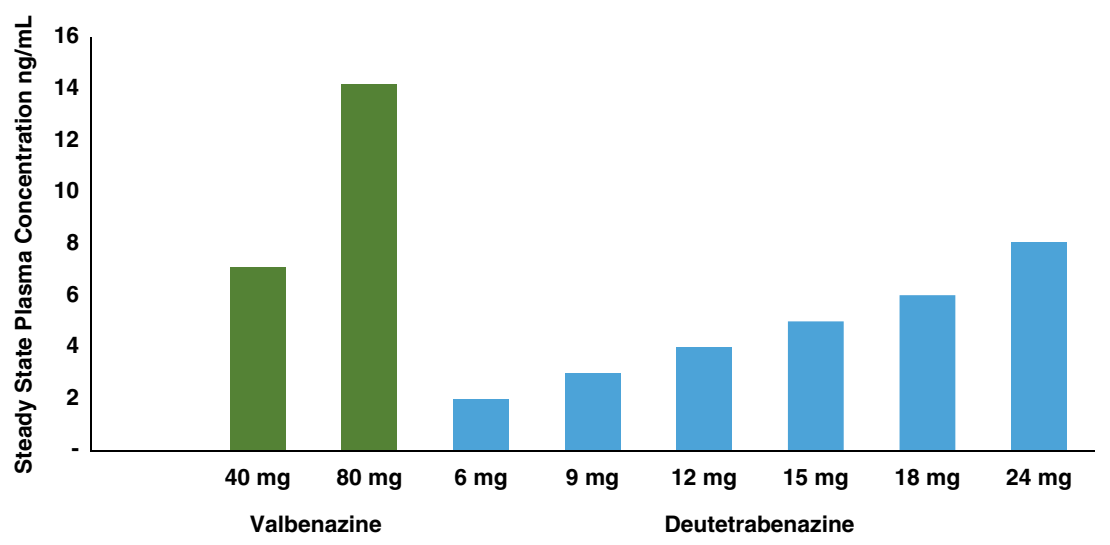

\section{Therapeutic Drug Levels Predict VMAT2 Occupancy Levels}

Since valbenazine is linked to the amino acid valine, then converted to just a single tetrabenazine isomer, whereas deutetrabenazine is converted into 4 active isomers (Figure 1), the doses administered (6-48 $\mathrm{mg}$ a day in divided doses for deutetrabenazine versus $40-80 \mathrm{mg}$ once per day for valbenazine) cannot be directly compared. Furthermore, blood levels of their respective active metabolite(s) cannot be directly compared since the active components of valbenazine and deutetrabenazine differ quite considerably from each other (Figures 1 and 2). The pharmacologic actions of these metabolites also differ a great deal (Table 1).

Instead, to compare the pharmacodynamics of valbenazine with deutetrabenazine, one can calculate and compare estimates of the degree of VMAT2 targeting over their respective dosage ranges. To do this, it is necessary first to quantify the amount(s) of active metabolite(s) present after administration of a given dose of each agent (Figures 2 and 3) and then to convert these blood levels into estimates (Table 2) of VMAT2 occupancies (Figure 4). From a pharmacological perspective, it is comparison of these VMAT2 occupancies that can potentially determine therapeutic differences between valbenazine and deutetrabenazine due to differential engagement of the therapeutic target VMAT2 at different doses (Figure 4).
The concentrations of the active components at VMAT2 for each agent $((+)$ dihydrotetrabenazine for valbenazine and the sum of both $(+)$ alpha and $(+)$ beta dihydrodeuterated tetrabenazine for deutetrabenazine) are shown across their respective dosing ranges in Figure 3 according to the available published data. $^{17-20}$ As already mentioned, the plasma drug levels of the active metabolite of valbenazine cannot be compared directly to those of deutetrabenazine in Figure 3, not only because the doses are different, but especially because only the $(+)$ alpha dihydro isomer is present after valbenazine administration, whereas both the $(+)$ alpha and the $(+)$ beta dihydro isomers are present after deutetrabenazine, with the $(+)$ beta form by contrast being the predominant active metabolite at VMAT2 for deutetrabenazine (Figure 1). ${ }^{17-20}$ The purpose of noting the plasma drug levels of these agents in Figure 3 is not to compare them, but to convert them to VMAT2 estimated occupancies so occupancies of the therapeutic target can be compared (Figure 4).

\section{VMAT2 Occupancy by Valbenazine and Deutetrabenazine}

The plasma drug levels shown in Figure 3 have been converted into calculated estimates of VMAT2 receptor occupancy in Figure 4 utilizing standard 


\section{BRAINSTORMS-Clinical Neuroscience Update}

Table 2. Assumptions made in calculating receptor occupancy levels from plasma drug levels and in vitro binding values

- Since deutetrabenazine has 4 metabolites with potential binding to VMAT2 receptor, a competitive binding model was applied:

$f_{\text {occupancy }}=\frac{\frac{L_{1}}{K d_{1}}+\frac{L_{2}}{K d_{2}}}{\frac{L_{1}}{K d_{1}}+\left(1+\frac{L_{2}}{K d_{2}}\right)}$

- Ki data for VMAT2 binding of active isomers was taken from Grigoriadis et al. ${ }^{14}$

Isomer proportion data was taken from Skor et al. ${ }^{17}$

- Protein binding levels of alpha-dihydrotetrabenazine and beta-dihydrotetrabenazine are assumed to be $68 \%$ and $63 \%$, respectively. ${ }^{19,20}$

- Binding affinities of deuterated isomers are assumed to be same as for non-deuterated isomers. ${ }^{15,16}$ Plasma-to-brain ratio is assumed to be 1 .

Plasma drug levels of active isomers of valbenazine and deutetrabenazine can be converted into estimates of receptor occupancy utilizing receptor binding affinities and standard formulae for competitive binding, with reasonable assumptions about protein binding and brain penetration of isomers.

pharmacologic formulae and reasonable assumptions about protein binding and brain penetration; these are listed in Table $2 .^{14-17}$ Utilizing this approach, net VMAT2 inhibition by valbenazine and deutetrabenazine at various doses can be both estimated and compared. Here is where any differences between valbenazine and deutetrabenazine can be seen in terms of engagement of the therapeutic target VMAT2 across their respective dose ranges (Figure 4). Of course, these are only estimates based upon the specific assumptions made (Table 2), but the relative differences between valbenazine and deutetrabenazine would be the same with different assumptions applied to both agents. Also, there is no estimate of the variance that would likely be present in a large population of TD patients, but the values in Figure 4 provide a rational basis for comparing the therapeutic activities of valbenazine and deutetrabenazine.

One can readily see that both agents cause a comparable and high degree of VMAT2 inhibition at their highest doses (eg, 91\% VMAT2 occupancy for valbenazine at the $80 \mathrm{mg}$ dose and $92 \%$ VMAT2 occupancy for deutetrabenazine at the $48 \mathrm{mg}$ dose) (Figure 4). However, the differences between the two agents become more marked at doses lower than this. Specifically, VMAT2 occupancy at the low dose of valbenazine $(40 \mathrm{mg})$ falls to $84 \%$ occupancy, whereas VMAT2 occupancies for deutetrabenazine at doses between $6 \mathrm{mg}$ and $42 \mathrm{mg}$ range between $51 \%$ and $92 \%$ (Figure 4 ).

\section{Beyond VMAT2 to 5 HT7 and D2}

Another pharmacologic differentiation between valbenazine and deutetrabenazine is that valbenazine has selective actions on VMAT2, whereas deutetrabenazine has additional actions on various monoamine receptors. To determine how much binding there is of deutetrabenazine isomers to these other receptors and thus whether this binding is likely to be clinically relevant, the plasma drug levels shown in Figure 3 can be converted into calculated estimates of serotonin, dopamine, and alpha noradrenergic receptor occupancies for the (-) isomers of deutetrabenazine (Table 1 and Figure 5) just as they were for VMAT2 occupancies in Figure 4. Calculations utilizing the same standard pharmacologic formulae and assumptions about protein binding and brain penetration listed in Table 2 and applied to VMAT2 occupancy calculations shown in Figure $4^{14-17}$ have been applied to other neurotransmitter receptor occupancy calculations shown in Figure 5. Here is where the differences between valbenazine and deutetrabenazine can be seen in terms of the degree of engagement of these additional monoamine receptors, with deutetrabenazine having actions there and valbenazine not having these actions. One can readily see that the serotonin 5HT7 receptor has notable occupancy by the active metabolites of deutetrabenazine (eg, over $50 \%$ at a single $15 \mathrm{mg}$ dose comparable to $30 \mathrm{mg}$ a day) (Figure 5) - a dose that generates $88 \%$ VMAT2 receptor occupancy (Figure 4). This same dose also generates over $20 \%$ occupancy of dopamine D2 receptors but negligible interaction with other neurotransmitter receptors (Figure 5).

These additional receptor actions clearly differentiate deutetrabenazine from valbenazine which lacks them, but are they clinically relevant? The D2 actions of deutetrabenazine at therapeutic dosing levels are likely to be below the threshold for notable physiological effects often estimated to require $60 \%$ D2 receptor occupancy or more. ${ }^{13}$ However, small amounts of D2 blockade could 


\section{BRAINSTORMS-Clinical Neuroscience Update}

Figure 4. Calculated VMAT2 receptor occupancy of both deutetrabenazine and valbenazine at Cmas. Plasma drug levels shown in Figure 3 can be converted into estimates of VMAT2 occupancy at various doses of both valbenazine and deutetrabenazine utilizing the binding affinities shown in Table 1, the assumptions shown in Table 2, and the proportion of each isomer shown in Figures 1 and 2. On the left (A) are the estimates of VMAT2 occupancy over the therapeutic dosing range of both compounds shown as smooth overlapping curves. On the right (B) are the actual calculated occupancies at each dose. Valbenazine dose is given once a day, and the various deutetrabenazine doses shown are given twice a day.
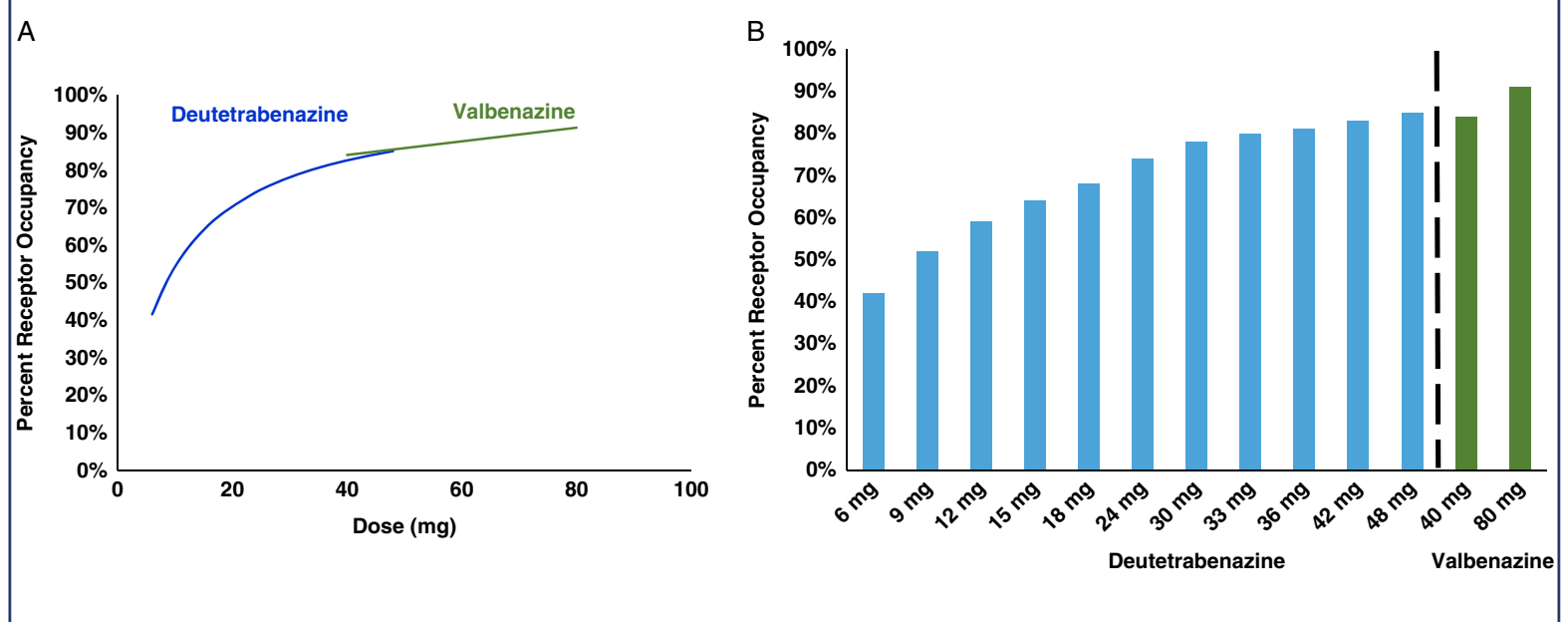

be additive to the action of concomitantly administered D2 antagonists and could either enhance their antipsychotic actions or increase their drug-induced parkinsonism. Also, it is likely that physiologically relevant actions at $5 \mathrm{HT} 7$ receptors are indeed present at the higher doses of deutetrabenazine (Figure 5). It is not well established what the clinical effects of 5HT7 antagonism would be, but several antidepressants and antipsychotics share this binding property, ${ }^{13}$ and in animal models, 5HT7 blockade enhances serotonin release, reduces circadian rhythm dysfunction, reduces negative motivation, and exerts procognitive and antidepressant actions (Figure 6). ${ }^{13}$ Whether these actions will accompany deutetrabenazine and not valbenazine remains unknown, but the potential therapeutic actions of 5HT7 occupancy might be worth further investigation. Actions at other monoamine receptors (eg, other serotonin receptors as well as alpha noradrenergic receptors) are unlikely to be physiologically relevant at therapeutic doses of deutetrabenazine.

\section{Threading the Needle}

Since the therapeutic target of both valbenazine and deutetrabenazine is VMAT2, the questions become what can we learn from the similarities and the differences in estimated VMAT2 binding properties of valbenazine versus deutetrabenazine and can these observations help determine potential advantages of one agent over the other for certain patients? The answers may depend upon how much VMAT2 inhibition is needed to "thread the needle" between mechanism-related side effects such as sedation and drug-induced parkinsonism from too much VMAT2 inhibition on the one hand and suboptimal therapeutic benefit for tardive dyskinesia movements from too little VMAT2 inhibition on the other hand. In addition to these considerations, numerous other pharmacologic variables could affect the ideal amount of VMAT2 inhibition for any given patient (see Figure 7). That is, since the therapeutic benefits of both valbenazine and deutetrabenazine are mediated by reducing the amount of dopamine acting at hypothetically supersensitive postsynaptic D2 dopamine receptors, ${ }^{1,2,13}$ not only is the concentration of VMAT2 inhibitor at synaptic vesicles in the presynaptic dopamine neuron important (modeled in Figures 2 and 3), and the extent to which this results in VMAT2 inhibition important (modeled in Figure 4), especially key is how much this VMAT2 inhibition reduces presynaptic dopamine concentration, and thus synaptic and postsynaptic dopamine (Figure 7). The amount of dopamine at supersensitive postsynaptic D2 receptors needed to reduce TD movements is not just dependent upon VMAT2 inhibition. It is also dependent upon several other factors: (1) the degree of supersensitivity of these postsynaptic D2 receptors; (2) whether the patient is also receiving a D2blocking antipsychotic drug (as is the case for most patients with TD); (3) the dose of that antipsychotic; 


\section{BRAINSTORMS-Clinical Neuroscience Update}

Figure 5. Calculated occupancy of deutetrabenazine at VMAT2 and monoamine receptors at $15 \mathrm{mg}$ dose. Plasma drug levels for the (-) isomers shown in Figure 2 at the $15 \mathrm{mg}$ dose of deutetrabenazine can be converted into estimates of occupancies of various monoamine receptors and compared to the occupancy of VMAT2 by the $(+)$ isomers shown in Figure 4 by utilizing the binding affinities shown in Table 1 and the assumptions shown in Table 2. Noted here is the moderate amount of serotonin 5HT7 receptor occupancy level, near the $60 \%$ threshold for physiologically relevant effects. Lower in amount is the D2 dopamine receptor occupancy, which is below the physiologically relevant level of occupancy to act on its own, but it may contribute to D2 dopamine actions of concomitantly administered antipsychotics. Other monoamine receptor binding is probably too low for clinical or physiological relevance.

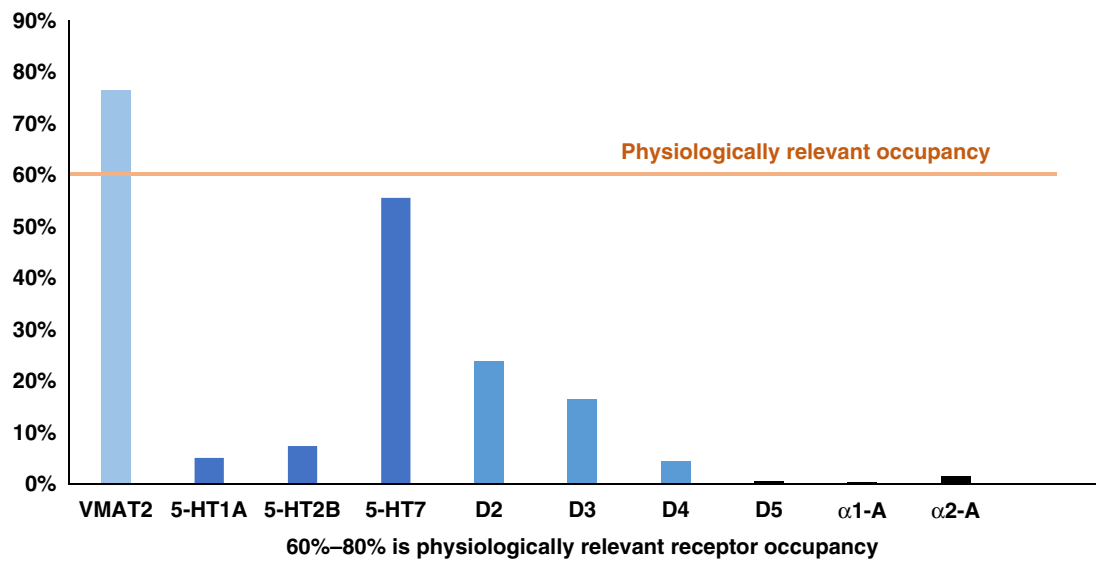

Figure 6. Hypothetical clinical outcomes of serotonin 5HT7 receptor blockade. Two isomers of deutetrabenazine have high affinity for $5 \mathrm{HT7}$ serotonin receptors. At therapeutic doses, deutetrabenazine likely has substantial occupancy of 5 HT7 receptors. Possible clinical relevance is unknown, but based on other drugs with 5 HT7 binding properties and preclinical studies, it could have procognitive and antidepressant properties. No adverse events are known to be associated with $5 \mathrm{HT} 7$ receptors.

$5 \mathrm{HT}_{7}$ Binding Affinity of Deutetrabenazine Isomers: - (-)-alpha-dihydro-deutetrabenazine $(\mathrm{Ki}=71 \mathrm{nM})$

- (-)-beta-dihydro-deutetrabenazine $(\mathrm{Ki}=6 \mathrm{nM})$

Possible Therapeutic Actions:

- Reduced circadian rhythm dysfunction

- Reduced negative symptoms

- Procognitive

- Antidepressant

Possible Adverse Events:

- Unknown

and (4) the affinity of that antipsychotic for D2 receptors. All these properties will be factors in determining to what extent any antipsychotic competes successfully with dopamine to occupy these same D2 receptors (Figure 7). One can thus expect that a broad range of VMAT2 inhibition may be required for the large and heterogeneous population of TD patients.

\section{Putting It All Together to Choose a VMAT2 Inhibitor}

In practice, looking at the therapeutic doses of VMAT2 inhibitors from published clinical trials, it seems that most patients will require a high degree ( $>90 \%$ ) of VMAT2 inhibition for the best balance between efficacy for TD and tolerability. ${ }^{5-12}$ In theory, it might not matter which agent you use to get this same degree of VMAT2 inhibition, but ease of use by giving the agent requiring less titration and only once a day dosing (valbenazine) might be preferred in the typical patient over the agent requiring more titration and twice daily dosing with food (deutetrabenazine).

How about patients who find the high degrees of VMAT2 occupancy at top doses of either agent too sedating or causing drug-induced parkinsonism? In these cases, it would be logical to lower the dose of VMAT2 inhibitor. As long as the single option for a lower dose with valbenazine is adequate, this is an option. However, if the loss of efficacy is too great with a $50 \%$ dose reduction, it might be better to explore the best dose from among the many more options with deutetrabenazine and titrate to the individual's need 


\section{BRAINSTORMS-Clinical Neuroscience Update}

Figure 7. Factors contributing to signal output from D2 dopamine receptors in tardive dyskinesia: potentially manageable by amount of VMAT2 inhibition. Shown here are the numerous pharmacologic variables that could affect the ideal amount of VMAT2 inhibition for any given patient (see Figure 5). Therapeutic benefits of both valbenazine and deutetrabenazine are thought to be mediated by reducing the amount of dopamine at hypothetically supersensitive postsynaptic D2 dopamine receptors. Thus, not only is the concentration of VMAT2 inhibitor at synaptic vesicles in the presynaptic dopamine neuron important, and the extent to which this results in VMAT2 inhibition, other especially key factors are how much this VMAT2 inhibition reduces presynaptic dopamine concentration, and thus synaptic and postsynaptic dopamine concentrations. Furthermore, the amount of dopamine at supersensitive postsynaptic D2 receptors is not just dependent upon VMAT2 inhibition but also upon the degree of supersensitivity of these postsynaptic D2 receptors. Additional factor to consider include whether the patient is also receiving a D2-blocking antipsychotic drug (as is the case for most patients with TD), the dose of that antipsychotic and the affinity of that antipsychotic for D2 receptors, since these properties of the antipsychotic drug will determine to what extent it competes successfully with dopamine to occupy these same D2 receptors.

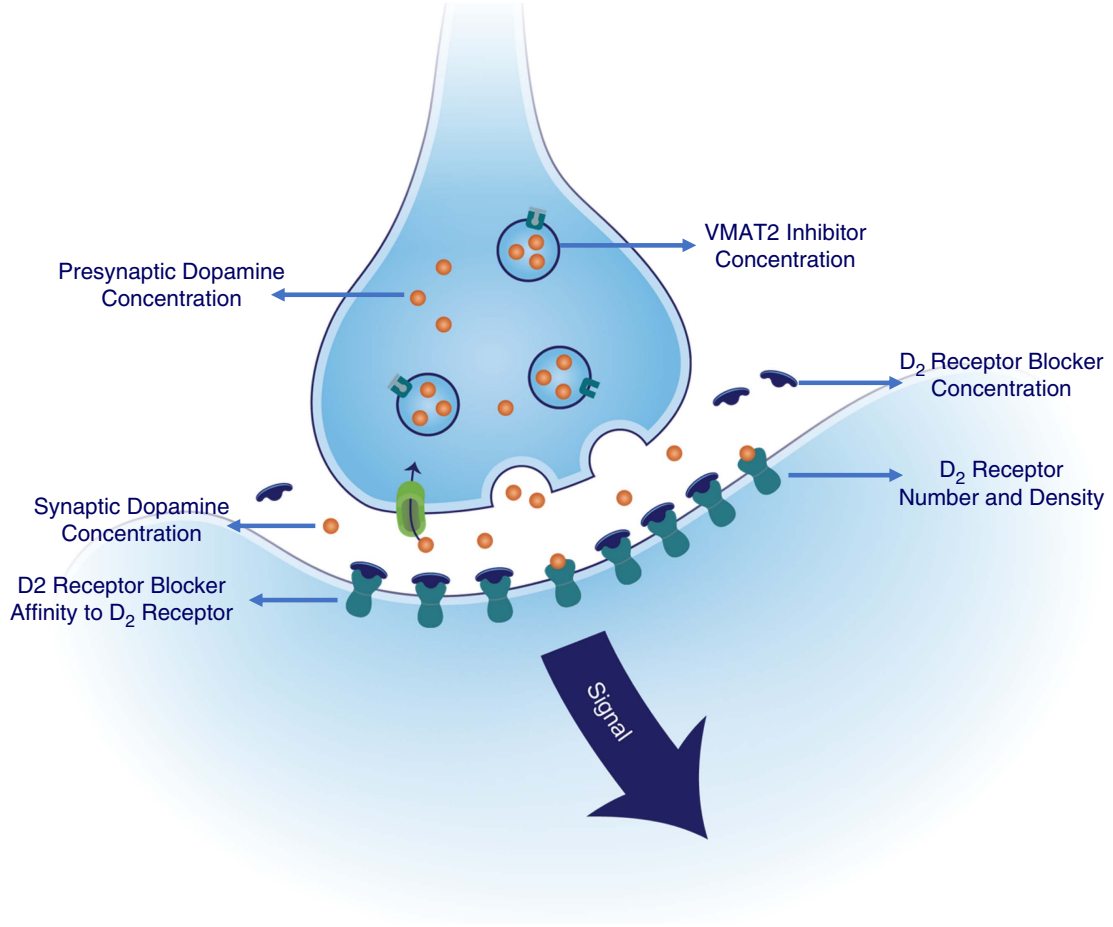

for balance between efficacy and tolerability. In fact, patients who require careful dose titration for any reason, including those who might require low levels of VMAT2 inhibition for any reason, would benefit from the multiple dosing options of deutetrabenazine.

\section{Summary and Conclusions}

Knowing how the VMAT2 inhibitors quantitatively engage their therapeutic targets across their dosing ranges can assist in selecting a specific agent for a specific patient. Valbenazine and deutetrabenazine both have comparable and high degrees of VMAT2 inhibition at their top doses. Deutetrabenazine but not valbenazine also binds serotonin $5 \mathrm{HT} 7$ receptors to a moderate degree and D2 dopamine receptors to a low degree, but the clinical significance of this is uncertain. Deutetrabenazine has more dosing options to titrate varying degrees of VMAT2 occupancy for those patients who require careful dosing titration, but a shorter half-life (9-20 hours), requiring twice a day administration with food. Valbenazine has fewer dosing options and less need for titration, yet a longer half-life (15-20 hours), allowing once a day administration.

\section{Acknowledgments}

The author would like to gratefully acknowledge the assistance of Rand Kittani, Miriam Cohen, PhD, and 


\section{BRAINSTORMS-Clinical Neuroscience Update}

Julia Dey, PhD, in preparing the figures, graphs, and calculations used in this article.

\section{References:}

1. Stahl SM. Neuronal traffic signals in tardive dyskinesia: not enough "stop" in the motor striatum. CNS Spectr. 2017; 22(6): 427-434.

2. Stahl SM. Mechanism of action of vesicular monoamine transporter 2 (VMAT2) inhibitors for tardive dyskinesia: reducing dopamine leads to less "go" and more "stop" from the motor striatum for robust therapeutic effects. CNS Spectr. 2018; 23(1): 1-6.

3. Bhidayasiri R, Fahn S, Weiner WJ, Gronseth GS, Sullivan KL, Zesiewicz TA. Evidence based guideline: treatment of tardive syndromes. Neurology. 2013; 81(5): 463-469.

4. Bhidayasiri R, Jitkritsandakul O, Friedman JH, Fahn S. Updating the recommendations for treatment of tardive syndromes: a systematic review of new evidence and practical treatment algorithm. J Neurol Sci. 2018; 389: 67-75.

5. Citrome L. Deutetrabenazine for tardive dyskinesia: a systematic review of the efficacy and safety profile for this newly approved novel medication - what is the number needed to treat, number needed to harm and likelihood to be helped or harmed? Int J Clin Pract. 2017; 71(11): e13030. doi: 10.1111/ijcp.13030.

6. Cummings MA, Proctor GJ, Stahl SM. Deuterium tetrabenazine for tardive dyskinesia. Clin Schizophr Relat Psychoses. 2018; 11(4): 214-220.

7. Fernandez HH, Factor SA, Hauser RA, et al. Randomized controlled trial of deutetrabenazine for tardive dyskinesia: the ARM-TD study. Neurology. 2017; 88(21): 2003-2010.

8. Citrome L. Tardive dyskinesia: placing vesicular monoamine transporter type 2 (VMAT2) inhibitors into clinical perspective. Expert Review of Neurotherapeutics. 2018; 18(4): 323-332. doi: 10.1080/14737175.2018.1455504.

9. Hauser RA, Factor SA, Marder SR, et al. KINECT 3: a phase 3 randomized, double-blind, placebo-controlled trial of valbenazine for tardive dyskinesia. Am J Psychiatry. 2017; 174(5): 476-484.
10. Freudenreich O, Remington G. Valbenazine for tardive dyskinesia. Clin Schizophr Relat Psychoses. 2017; 11(2): 113-119.

11. Niemann N, Jankovic J. Treatment of tardive dyskinesia: a general overview with focus on the vesicular monoamine transporter 2 inhibitors. Drugs. 2018; 78(5): 525-541.

12. Scorr LM, Factor SA. VMAT2 inhibitors for the treatment of tardive dyskinesia. J Neurol Sci. 2018; 389: 43-47.

13. Stahl SM. Stahl's Essential Psychopharmacology: Neuroscientific Basis and Practical Application, 4th ed. Cambridge, UK: Cambridge University Press; 2013.

14. Grigoriadis DE, Smith E, Hoare SRJ, Madan A, Bozigian H. Pharmacologic characterization of valbenazine and its metabolites. J Pharmacol Exp Ther. 2017; 361(3): 454-461.

15. Gant TG. Using deuterium in drug discovery: leaving the label in the drug. J Med Chem. 2014; 57(9): 3395-3611.

16. Stamler D, Bradbury M, Brown F. The pharmacokinetics and safety of deuterated tetrabenazine. Neurology. 2013; 80(7 Suppl): P07.210.

17. Skor H, Smith EB, Loewen G, O'Brien CF, Grigoriadis DE, Bozigian $\mathrm{H}$. Differences in dihydrotetrabenazine isomer concentrations following administration of tetrabenazine and valbenazine. Drugs R D. 2017; 17(3): 449-459.

18. Derangula VR, Pilli NR, Nadavala SK, Adireddy V, Inanadugu JK, Ponneri V. Liquid chromatography-tandem mass spectrometric assay for the determination of tetrabenazine and its active metabolites in human plasma: a pharmacokinetic study. Biomed. Chromatogr. 2013; 27(6): 792-801.

19. INGREZZA (valbenazine) Clinical Pharmacology. US Food \& Drug Administration. Center for Drug Evaluation and Research. Clinical pharmacology and biopharmaceutics review(s). INGREZZA. May 2017. https:/ / www.accessdata.fda.gov/ drugsatfda_docs/nda/2017/209241oirg1s000clinpharmr.pdf (accessed June 1, 2018).

20. AUSTEDO (deutetrabenazine) Clinical Pharmacology. US Food \& Drug Administration. Center for Drug Evaluation and Research. Clinical pharmacology and biopharmaceutics review(s). AUSTEDO. May 2017. https://www.accessdata.fda.gov/ drugsatfda_docs/nda/2017/208082orig1s000clinpharmr.pdf (accessed June 1, 2018). 